\title{
Oral Nifedipine versus Intravenous Labetalol in Hypertensive Emergencies of Pregnancy- A Randomised Trial
}

\author{
Subiya Jamil ${ }^{1}$, Mallika Sengupta르, Chhandos Saha ${ }^{3}$, Dipanjan Bandhya ${ }^{4}$, Rajesh De ${ }^{5}$, Manjir Mitra ${ }^{6}$, Debdatta Ghosh ${ }^{7}$ \\ ${ }^{1}$ Department of Obstetrics and Gynaecology, Malbazar Super-Speciality Hospital, Malbazar, West Bengal, India. \\ ${ }^{2}$ Department of Obstetrics and Gynaecology, R.G. Kar Medical College and Hospital, Kolkata, West Bengal, India. \\ ${ }^{3}$ Department of Obstetrics and Gynaecology, R.G. Kar Medical College and Hospital, Kolkata, West Bengal, India. \\ ${ }^{4}$ Department of Obstetrics and Gynaecology, R.G. Kar Medical College and Hospital, Kolkata, West Bengal, India. \\ ${ }^{5}$ Department of Community Medicine, R.G. Kar Medical College and Hospital, Kolkata, West Bengal, India. \\ ${ }^{6}$ Department of Obstetrics and Gynaecology, Baranagar State General Hospital, Kolkata, West Bengal, India. \\ ${ }^{7}$ Department of Obstetrics and Gynaecology, R.G. Kar Medical College and Hospital, Kolkata, West Bengal, India.
}

\section{ABSTRACT}

\section{BACKGROUND}

Hypertensive disorders during pregnancy are the most significant and unresolved problems in obstetrics. Antihypertensive treatment is indicated for all pregnant women with blood pressure greater than or equal to $160 \mathrm{mmHg}$ systolic or 110 mmHg diastolic. This degree of hypertension requires urgent assessment and management. In this study, we compared the effectiveness of oral nifedipine and intravenous labetalol in the control of hypertensive emergencies of pregnancy.

\section{METHODS}

In this open-label randomised trial, 60 pregnant women with $\geq 28$ weeks of gestation with sustained severe hypertension (systolic blood pressure of $\geq 160$ or/and diastolic blood pressure $\geq 110 \mathrm{mmHg}$ ) were allocated to two groups in a randomised manner. One group $(n=30)$ received intravenous labetalol and the other group $(n=30)$ received oral nifedipine according to National Health Mission, Government of India protocol. Injection labetalol was given in repeated doses (20, $40,80,80$, and 80 ) every 10 minutes and oral nifedipine capsule was repeated in 10 $\mathrm{mg}$ doses at 30 minutes interval until the target $\mathrm{BP}$ of $\leq 150 / 100 \mathrm{mmHg}$ was achieved.

\section{RESULTS}

The result of this study showed that after 30 minutes, more patients in the nifedipine group (66.6\%) achieved the target blood pressure as compared to the labetalol group (46.6\%). The mean time taken to achieve this target blood pressure was $30.6 \pm 7.8$ minutes versus $34 \pm 7.7$ minutes for nifedipine and labetalol group respectively $(\mathrm{p}=0.09)$.

\section{CONCLUSIONS}

Both oral nifedipine and intravenous labetalol regimens are equally effective and well tolerated when used for rapid control of blood pressure in severe hypertension of pregnancy. Nifedipine may be preferred due to lesser repetition of doses, simple and flat dosage pattern, and ease of oral administration, low cost and wide availability.

\section{KEY WORDS}

Hypertension, Labetalol, Nifedipine, Pregnancy
Corresponding Author: Dr. Mallika Sengupta, RMO Quarters, Block B, Flat 1,

$R$ G Kar Medical College and Hospital,

1, Khudiram Bose Sarani

Kolkata-700004, West Bengal, India.

E-mail: drmallikasengupta@gmail.com

DOI: $10.14260 /$ jemds/2019/775

Financial or Other Competing Interests: None.

How to Cite This Article:

Jamil S, Sengupta M, Saha C, et al. Oral nifedipine versus intravenous labetalol in hypertensive emergencies of pregnancy- $a$ randomised trial. J. Evolution Med. Dent. Sci. 2019;8(48):3588-3592, DOI: 10.14260/jemds/2019/775

Submission 03-10-2019,

Peer Review 13-11-2019,

Acceptance 20-11-2019,

Published 02-12-2019. 


\section{BACKGROUND}

Hypertensive disorders complicate roughly 5 - $10 \%$ of pregnancies worldwide and are considered to be one of the leading causes of maternal and perinatal morbidity and mortality. ${ }^{1}$ According to a study, the prevalence of hypertensive disorders was $7.8 \%$ with preeclampsia in $5.4 \%$ of study population in India. ${ }^{2}$ Hypertensive disorders of pregnancy have contributed to $10 \%$ to $15 \%$ of direct maternal deaths globally and majority of these deaths occur in the low and middle-income countries. ${ }^{3}$ Although the outcome for most of these pregnancies is good, women with severe hypertension have an increased risk of developing end organ damage such as acute renal failure, hepatic failure, abnormalities of the clotting system, pulmonary oedema, intracerebral haemorrhage, stroke, acute myocardial infarction, eclampsia and hypertensive encephalopathy as well as adverse perinatal outcome like intrauterine growth retardation, intrauterine death. ${ }^{4}$ Pregnant women with severe hypertension should receive anti-hypertensive therapy to lower their blood pressure rapidly but in a controlled manner as rapid reduction of blood pressure can result in hypo perfusion of vital organs (brain, heart). Recent guidance from National Institute of Health and Clinical Excellence, UK, recommends inpatient treatment of severe hypertension of pregnancy with labetalol (Oral or Intravenous), intravenous hydralazine or oral nifedipine as first-line alternative antihypertensives within the critical care settings. ${ }^{5}$

Presently the decision of drug selection depends on the clinical experience of the obstetrician. Furthermore, the decision is also influenced by ease of administration, the cost of the drug and what is known about the adverse effects. Hydralazine had been the drug of choice for a long time; however, the faith in hydralazine has decreased over the past decade due to evidence of increased maternal and foetal complications associated with its use. ${ }^{6}$ Currently, there are few trials that have compared nifedipine and labetalol in terms of control of severe hypertension during pregnancy. Therefore, their comparative benefits and side effects remain uncertain.

So, our aim in this study was to compare the efficacy and safety of oral nifedipine and intravenous labetalol in the control of severe pregnancy induced hypertension.

\section{METHODS}

We performed an open-label randomised trial in pregnant patients suffering from hypertensive emergencies. This trial was conducted in Dept. of Obstetrics and Gynaecology, RG Kar Medical College and Hospital, India. Recruitment took place between 1st July 2016 to 30th June 2017. Pregnant women carrying single viable foetus at $\geq 28$ wks. of gestation with sustained severe hypertension were approached for enrolment in the trial. Sustained severe hypertension was defined as systolic BP $\geq 160 \mathrm{mmHg}$ or/and diastolic BP $\geq 110$ $\mathrm{mmHg}$ for at least two occasions in the last 4 hours at least 20 minutes apart. The latest blood pressure reading prior to enrolment must fulfil the criteria of severe hypertension. Other inclusion criteria were medical decision to rapidly control blood pressure, acceptable cardiotocograph (CTG) and maternal heart rate between $\geq 60$ and $<120 \mathrm{bpm}$.

Women with history of cardiac arrhythmia, heart failure, asthma, allergy to either nifedipine or labetalol, nonpregnancy related hypertension and any antihypertensive treatment in the preceding 72 hours were excluded from the study. Women who agreed to participate provided written informed consent. The study was approved by the medical ethics committee of RG Kar Medical College.

Sample size was calculated based on the study result of Vermillion et $\mathrm{al}^{7}$ showing nifedipine group to achieve target blood pressure with significant rapidity than labetalol group $(25 \pm 13.6$ minutes versus $43.6 \pm 25.4$ minutes, $p=0.002)$.Power of the study was taken as $80 \%$ and level of significance $5 \%$, sample size of 30 for each group was calculated. Block randomization was performed with 1:1 allocation with randomly selected block sizes of 2 and 4 by an independent person using Excel 2007.

Participants were allowed to rest in bed in a semi recumbent position with history taking, clinical examination and relevant investigations. The blood pressure was measured, preferably in sitting position with mercury sphygmomanometer as per standard recommendation. Korotkoff sounds I (the first sound) and V (the disappearance of sound) was used to denote the systolic blood pressure and diastolic blood pressure respectively. The BP was measured every 10 minutes for at least 60 minutes or more until the target blood pressure was achieved.

One group $(n=30)$ received intravenous labetalol and the other group $(n=30)$ received oral nifedipine according to this study protocol. Intravenous labetalol injection was given in an escalating dose regimen $(20,40,80,80$, and $80 \mathrm{mg})$ every 10 minutes and oral nifedipine capsule was repeated in 10 mg doses at 30 minutes interval until the target BP of $\leq$ $150 / 100 \mathrm{mmHg}$ was achieved. Crossover treatment was planned after non-achievement of target blood pressure. Primary outcome measure was time taken to achieve blood pressure $\leq 150 / 100 \mathrm{mmHg}$ and secondary outcome measures were total antihypertensive doses needed to achieve target blood pressure, management at the end of the trial(expedited or expectant),mode of delivery, side effects of antihypertensive, maternal and perinatal complications. The patients were monitored for occurrence of any complications during antenatal, intranatal \& post-natal period. The mode of delivery, neonatal \& maternal outcome had been noted. Where the treatment was completed, participants were interviewed to assess the acceptability of the method assigned.

After the successful control of blood pressure further antihypertensive therapy was started 2 hours after the last trial medication and delivery of the baby as the definitive treatment for severe pregnancy-induced hypertension was started for participants at or near term, as standard practice. Stabilised patients who were managed expectantly were sent to the normal ward for further observation.

Primary outcome measure was time taken to achieve target blood pressure of $\leq 150 / 100 \mathrm{mmHg}$ and secondary outcome measures were total antihypertensive doses needed to achieve target blood pressure, management at the end of the trial (expedited or expectant), mode of delivery, side effects of antihypertensives, maternal and perinatal complications. 


\section{Statistical Analysis}

Analysis was based on the intention to treat principle. The normal distribution of continuous data was checked with the one-sample Kolmogorov-Smirnov test. Continuous data was analyzed with Student's unpaired t-test or Mann-Whitney U test where indicated. Categorical $2 \times 2$ data sets were analyzed with Fisher exact test. A repeated measure analysis of the variance (ANOVA) was applied to repeated measures of blood pressure. All tests were two sided and $\mathrm{P}<0.05$ was taken as the level of significance.

\section{RESULTS}

Repeated measures analysis of variance indicated that both systolic $(\mathrm{F}=146.732, \mathrm{p}=<0.001$ and $\mathrm{F}=146.750, \mathrm{p}=<0.001$ for nifedipine and labetalol group respectively) and diastolic $(\mathrm{F}=241.304, \quad \mathrm{p}=<0.001$ and $\mathrm{F}=150.520, \quad \mathrm{p}=<0.001$ for nifedipine and labetalol group respectively) blood pressure significantly decreased over time and from 20 minutes onward from the initiation of the trial, the decrease of mean blood pressure, both systolic and diastolic, was significantly more in the nifedipine $\operatorname{group}(\mathrm{p}=<0.05)$. Number of doses needed in the oral nifedipine group (1.3 \pm 4.7$)$ was significantly lower $(\mathrm{p}=0.0001)$ than the intravenous labetalol group (3.4 7.7). Cumulative urine output gradually increased over time for the nifedipine group $(p=0.0001)$. Crossover treatment was not required. No maternal or foetal adverse outcome was noted.

\begin{tabular}{|c|c|c|c|}
\hline Characteristics & $\begin{array}{c}\text { Nifedipine } \\
(n=30)\end{array}$ & $\begin{array}{c}\text { Labetalol } \\
(\mathrm{n}=\mathbf{3 0})\end{array}$ & $\mathbf{p}$ \\
\hline Age (Years) $\bullet$ & $21.2 \pm 5.2$ & $20.86 \pm 4.2$ & 0.744 \\
\hline Gravidity† & $1.6(1-4)$ & $1.7(1-4)$ & 0.2026 \\
\hline Parity† & $0(0-3)$ & $1(0-3)$ & 0.392 \\
\hline Gestation (weeks) • & $35.5 \pm 2.08$ & $36.06 \pm 1.87$ & 0.277 \\
\hline BMI $\left(\mathrm{kg} / \mathrm{m}^{2}\right) \bullet$ & $21.69 \pm 3.01$ & $22.76 \pm 2.72$ & 0.122 \\
\hline SBP Prior to Starting Treatment $(\mathrm{mmHg}) \bullet$ & $170.2 \pm 9.13$ & $167.53 \pm 7.53$ & 0.219 \\
\hline DBP Prior to Starting Treatment $(\mathrm{mmHg}) \bullet$ & $117.2 \pm 6.02$ & $117.3 \pm 5.9$ & 0.6242 \\
\hline Heart Rate (beats/minute) & $84 \pm 6.1$ & $85.5 \pm 7.4$ & 0.259 \\
\hline Proteinuriał & $11(37 \%)$ & $10(33.3 \%)$ & 0.814 \\
\hline Premonitory Signs of Eclampsiał & $4(13.3 \%)$ & $5(16.6 \%)$ & 0.5525 \\
\hline \multicolumn{4}{|c|}{$\begin{array}{c}\text { Table 1. Demographic and Clinical Characteristics of } \\
\text { Participants at Enrolment }\end{array}$} \\
\hline
\end{tabular}

\begin{tabular}{|c|c|c|c|c|}
\hline \multicolumn{2}{|c|}{ Outcome at the End of the Trial } & $\begin{array}{l}\text { Nifedipine } \\
(n=30)\end{array}$ & $\begin{array}{c}\text { Labetalol } \\
(n=30)\end{array}$ & $\mathbf{p}$ \\
\hline \multicolumn{2}{|c|}{$\begin{array}{l}\text { Time Taken to Reduce BP } \leq 150 / 100 \mathrm{mmHg} \text { (in min.) } \\
{[\text { [Mean } \pm \text { SD] }}\end{array}$} & $30.6 \pm 7.8$ & $34 \pm 7.7$ & 0.09 \\
\hline \multicolumn{2}{|c|}{$\begin{array}{l}\text { Total Antihypertensive Doses needed to Achieve the } \\
\text { Target BP [Mean } \pm \text { SD] }\end{array}$} & $1.3 \pm 0.47$ & $3.4 \pm 0.77$ & 0.0001 \\
\hline \multirow{2}{*}{$\begin{array}{l}\text { Management at the END of } \\
\text { the Trial [No (\%)] }\end{array}$} & Expedited delivery & $23(76.66 \%)$ & $24(80 \%)$ & \multirow{2}{*}{0.635} \\
\hline & Expectant management & $7(23.33 \%)$ & $6(20 \%)$ & \\
\hline \multirow{2}{*}{$\begin{array}{l}\text { Mode of Delivery } \\
\text { [No (\%)] }\end{array}$} & Caesarean section & $19(63.3 \%)$ & $16(53.3 \%)$ & \multirow{2}{*}{0.197} \\
\hline & Vaginal & 11(36.6\%) & $14(46.6 \%)$ & \\
\hline \multirow{6}{*}{$\begin{array}{l}\text { Maternal Side Effects } \\
\quad[\mathrm{No}(\%)]\end{array}$} & Nausea & $1(3.3 \%)$ & $2(6.6 \%)$ & 0.33 \\
\hline & Vomiting & $2(6.6 \%)$ & $2(6.6 \%)$ & 1 \\
\hline & Dizziness & $2(6.6 \%)$ & $3(10 \%)$ & 0.61 \\
\hline & Palpitation & $2(6.6 \%)$ & 0 & \\
\hline & Headache & $2(6.6 \%)$ & $1(3.3 \%)$ & 0.33 \\
\hline & Chest pain & 0 & $1(3.3 \%)$ & \\
\hline \multicolumn{2}{|c|}{ Maternal ICU Admission [No (\%)] } & $2(6.6 \%)$ & $3(10 \%)$ & 0.754 \\
\hline \multirow{4}{*}{$\begin{array}{l}\text { Neonatal Complications } \\
{[\text { No (\%)] }}\end{array}$} & $\begin{array}{l}\text { Neonatal ICU Care [No } \\
(\%)]\end{array}$ & $6(20 \%)$ & $7(23.35 \%)$ & 0.754 \\
\hline & Birth Weight < $2.5 \mathrm{Kg}$ & $4(13.3 \%)$ & $5(16.65 \%)$ & 0.55 \\
\hline & Perinatal Asphyxia & $2(6.6 \%)$ & $3(10 \%)$ & 0.754 \\
\hline & $\begin{array}{l}\text { APGARScore }<7 \text { At } 5 \\
\text { Mins. }\end{array}$ & $4(13.3 \%)$ & $6(20 \%)$ & 0.253 \\
\hline \multicolumn{5}{|c|}{ Table 2. Outcome at the End of Trial } \\
\hline \multicolumn{5}{|c|}{$\begin{array}{l}\text { Fisher exact test done for categorical variables, unpaired student's t test done for } \\
\text { mean and SD comparison. }\end{array}$} \\
\hline
\end{tabular}

\begin{tabular}{|c|c|c|c|c|}
\hline $\begin{array}{c}\text { Time from Treatment } \\
\text { Initiation (in Mins.) }\end{array}$ & $\begin{array}{c}\text { Nifedipine } \\
{[\mathbf{n = 3 0 ]}}\end{array}$ & $\begin{array}{c}\text { Labetalol } \\
{[\mathbf{n = 3 0 ]}}\end{array}$ & $\begin{array}{c}\mathbf{p} \\
\text { Value }\end{array}$ & $\begin{array}{c}\text { Chi } \\
\text { Square }\end{array}$ \\
\hline 10 & $0 \%$ & $3.3 \%$ & 0.244 & 1.354 \\
\hline 20 & $26.6 \%$ & $10 \%$ & 0.002 & 9.091 \\
\hline 30 & $66.6 \%$ & $46.6 \%$ & 0.006 & 7.364 \\
\hline 40 & $100 \%$ & $100 \%$ & & \\
\hline 50 & $100 \%$ & $100 \%$ & & \\
\hline 60 & $100 \%$ & $100 \%$ & & \\
\hline Table 3. Cumulative Percentage of the Subjects Achieving \\
Target BP Over Time \\
\hline
\end{tabular}

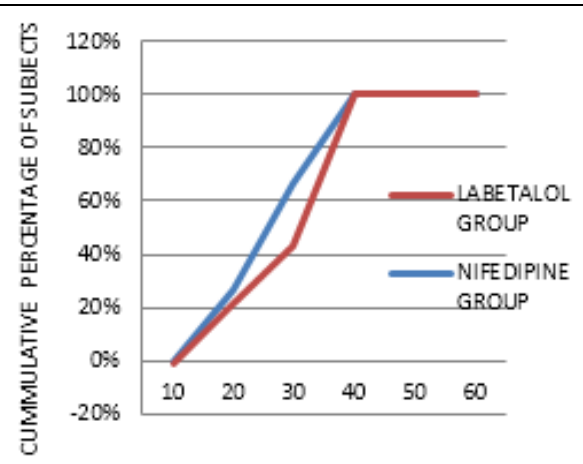

Cumulative Percentage of Subjects Who Achieved Blood Pressure $\leq 150 / 100 \mathrm{mmHg}$ Over Time

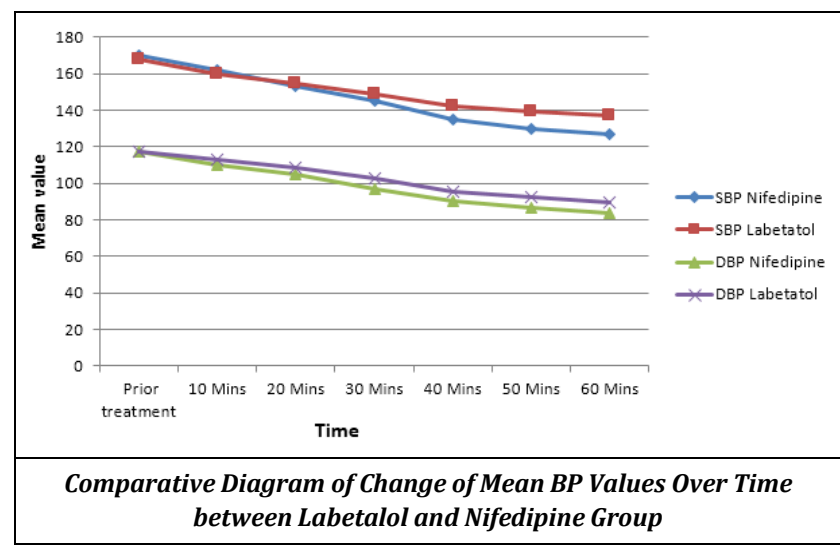

\section{DISCUSSION}

Our data indicate that both oral nifedipine and intravenous labetalol regimens are effective in controlling severe pregnancy induced hypertension, consistent with the reports of previous studies. The groups were similar at enrolment with respect to maternal age, parity, mean gestational age, BMI, and mean systolic and diastolic blood pressure, presence of proteinuria, and heart rate pattern. Majority of patients in both the groups belong to the age group of 16 to 20 years signifying prevalence of pregnancy induced hypertension in teen age pregnancy. Majority of the participants were primigravid (56\% in nifedipine group and $46 \%$ in labetalol group) and at $<37$ weeks of gestation $(70 \%$ in nifedipine group and $60 \%$ in labetalol group).

\section{Mean Time to Achieve Target Blood Pressure}

In this study pregnant women allocated to oral nifedipine group achieved target blood pressure more rapidly (expressed as mean \pm SD, 30.6 \pm 7.8 minutes) compared to intravenous labetalol group (34 \pm 7.7 minutes). However, no significant difference was found between the two groups 
$(\mathrm{p}=0.09)$. Our findings of nifedipine being equally effective as labetalol are in keeping with the findings of Raheem et $\mathrm{al}^{8}$ and Mukherjee at $\mathrm{al}^{9}$. In the study by Raheem et al mean time needed to achieve target blood pressure was $46 \pm 30$ minutes and $54 \pm 42$ minutes $(\mathrm{p}=0.45)$ in the nifedipine and labetalol group respectively. Mukherjee et al in their study showed the mean time needed to achieve target blood pressure was 43 \pm 16.7 minutes and $38.67 \pm 19.43$ minutes $(p>0.05)$ in the nifedipine and labetalol group respectively and the shorter time needed to achieve target blood pressure in the nifedipine group in my study may be explained by our standard dosing protocol $110 \mathrm{mg}$ oral nifedipine every 30 minutes) in comparison to their low dose protocol (5 mg oral nifedipine every 30 minutes). Repeated measures analysis of variance indicated that in the first hour both systolic $(\mathrm{F}=146.732, \quad \mathrm{p}=<0.001$ and $\mathrm{F}=146.750, \quad \mathrm{p}=<0.001$ for nifedipine and labetalol group respectively) and diastolic ( $\mathrm{F}=241.304, \quad \mathrm{p}=<0.001$ and $\mathrm{F}=150.520, \quad \mathrm{p}=<0.001$ for nifedipine and labetalol group respectively) blood pressure significantly decreased. From 30 minutes onward from the initiation of the trial, the between-group comparison showed a significant decrease of mean blood pressure (Both systolic and diastolic) in the nifedipine group compared to labetalol $\operatorname{group}(\mathrm{p}=<0.05)$. Vermillion et al. as well as Shekhar et al ${ }^{10}$ found nifedipine to be more efficacious than labetalol over the time similar to this study.

\section{Success Rate}

In this study we have found $100 \%$ success rate in achieving target blood pressure with both nifedipine and labetalol. No crossover treatment was required. Vermilion et al got similar results like this study, whereas Raheem et al reported $20 \%$ failure rate with both drugs and required crossover treatment.

\section{Number of Doses}

In this study the average doses of the trial medicine needed to reach the target blood pressure was $1.3 \pm 0.47$ (mean \pm SD)for nifedipine group and $3.4 \pm 0.77$ (mean \pm SD) for labetalol group, $\mathrm{P}$ value $=0.001$ indicated significant difference. This finding was consistent with the findings of Sekhar et al and Vermilion et al. Raheem et al and Mukerjee et al found no significant difference in this regard. The study may be questioned for using labetalol at 10 minutes interval instead of 15 minutes or 20 minutes interval as recommended by few studies. We chose 10 minutes dosing interval for intravenous labetalol and 30 minutes for oral nifedipine in accordance with our institutional protocol. This study used different dosing interval for nifedipine and labetalol group (30 minutes versus 10 minutes respectively) similar to the study by Mukherjee et al. All other trials used equal dosing interval for both the groups.

\section{Heart Rate}

Concerns have been raised about the safety of nifedipine in obstetric patients due to possibility of tachycardia with short acting nifedipine capsules. In this study mean maternal heart rate at the initiation of treatment was $84 \pm 6.1$ beats per minute in nifedipine group and $86 \pm 7.4$ in the labetalol group. Sixty minutes after initiations of treatment tachycardia was noted in nifedipine group (mean \pm SD, 108 \pm 4.8 ) and $72 \pm 8.7$ beats per minutes in labetalol group, $p=0.001$ showing significant differences over time but non-reassuring foetal heart rate pattern in cardiotocography was not observed in any of the patients in the nifedipine group. Similar findings were reported by Raheem et al in their study where mean maternal pulse rate was increased significantly over the first hour after treatment with nifedipine. Mukherjee et al found no significant difference in maternal heart rate as they have used low dose nifedipine ( $5 \mathrm{mg}$ in every $30 \mathrm{mins}$ ).

\section{Side Effects}

The common side effects in both the groups were nausea, vomiting, dizziness, palpitation, headache, and chest pain. Palpitation was present in only 2 patients in the Nifedipine group. Rests of the side effects were similar. This is consistent with the observations of many studies. Mukherjee et al found no complaint of palpitation in the nifedipine group, may be due to their low dose protocol.

\section{Magnesium Sulphate Injection and Nifedipine}

Magnesium sulphate infusion as seizure prophylaxis is indicated in women with severe pre-eclampsia as supported by favourable data from the Magpie trial ${ }^{11}$. In our study four patients $(13.3 \%)$ in the nifedipine group and five patients $(16.6 \%)$ in the labetalol group needed magnesium sulphate prophylaxis. No serious adverse events like severe hypotension or neuromuscular blockade was noticed with concurrent use of nifedipine and magnesium sulphate in this study.

\section{Urine Output}

In this study the cumulative urine output gradually increased over time for the nifedipine group and the difference being statistically significant $(\mathrm{p}=0.0001)$.Similar finding was shown by Vermillion et al and Raheem et al and Dhali et al. ${ }^{12}$

\section{Management at the End of the Trial}

After stabilization of the patient, 23 patients $(76.6 \%)$ of the nifedipine group and 24 patients $(80 \%)$ of the labetalol group had an expedited management $(p=0.6)$ and it indicates that expectant management has very little role in severe pregnancy induced hypertension. Rate of caesarean section was more than that of vaginal delivery, 19 patients $(63.3 \%)$ in nifedipine group and 16 patients (53.3\%) in the labetalol group and $(\mathrm{p}=0.19)$ showing no significant difference. The indications of caesarean sections were mostly induction failure, intrauterine growth retardation with oligohydramnios and in few due to appearance of premonitory symptoms of pre-eclampsia with unfavourable cervix ( 4 patients in nifedipine group and 5 patients in labetalol group, who received injection magnesium sulphate prophylaxis also).

\section{Maternal Complications}

No patients in this study developed eclampsia, overshoot hypotension, pulmonary oedema, hypertensive encephalopathy, stroke, acute myocardial infarction or acute renal failure. Only 2 patients (7\%) in nifedipine group and 3 patients $(10 \%)$ in labetalol group needed intensive care unit (ICU) admission due to delayed recovery from anaesthesia after caesarean section. 


\section{Neonatal Complications}

Occurrence of perinatal asphyxia, meconium staining of liquor, APGAR Score $<7$ at 5 minutes, low birth weight babies and need of neonatal care unit admission were similar in both the groups.

\section{CONCLUSIONS}

We found that both nifedipine and labetalol regimens are effective and well tolerated when used for the rapid control of blood pressure in severe hypertension of pregnancy. Though lowering of blood pressure over time was significantly more in the nifedipine group, we need further trial comprising of a larger sample size to reach the conclusion that oral nifedipine is efficacious than intravenous labetalol in hypertensive emergencies of pregnancy. The number of doses needed to reduce BP to $150 / 100 \mathrm{mmHg}$ was significantly less for the nifedipine group as compared to the labetalol group. The maternal and foetal side effect profile was similar for both the groups. Though maternal heart rate was significantly increased over time with the nifedipine regimen, this finding was not alarming as it caused only a milder degree of palpitation in few patients who recovered shortly after treatment. Also, urine output was found to increase significantly with nifedipine regimen. If only a choice has to be made, oral nifedipine may be preferred as it has a simple and flat dosage pattern, is an oral regimen, is low cost and is widely available..

\section{REFERENCES}

[1] Khan KS, Wojdyla D, Say L, et al. WHO analysis of causes of maternal death: a systematic review. Lancet 2006;367(9516):1066-74.

[2] Sajith M, Nimbargi V, Modi A, et al. Incidence of pregnancy-induced hypertension and prescription pattern of antihypertensive drugs in pregnancy. International Journal of Pharma Sciences and Research 2014;5(4):163-70.
[3] Duley L. The global impact of preeclampsia and eclampsia. Semin Perinatol 2009;33(3):130-7.

[4] Buchbinder A, Sibai BM, Caritis S, et al. Adverse perinatal outcomes are significantly higher in severe gestational hypertension than in mild preeclampsia. Am J Obstet Gynecol 2002;186(1):66-71.

[5] National Institute of Health and Clinical Excellence. Hypertension in Pregnancy, The management of hypertensive disorders during pregnancy. Clinical guidelines CG107 Issued: August 2010. Accessible on http://guidance.nice.org.uk/CG107. Last accessed 26 December 2010.

[6] Magee LA, Cham C, Waterman EJ, et al. Hydralazine for treatment of severe hypertension in pregnancy: metaanalysis. BMJ 2003;327(7421):955-60.

[7] Vermillion ST, Scardo JA, Newman RB, et al. A randomized, double-blind trial of oral nifedipine and intravenous labetalol in hypertensive emergencies of pregnancy. Am J Obstet Gynecol 1999;181(4):858-61.

[8] Raheem IA, Saaid R, Omar SZ, et al. Oral nifedipine versus intravenous labetalol for acute blood pressure control in hypertensive emergencies of pregnancy: a randomized trial. BJOG 2012;119(1):78-85.

[9] Mukherjee $S$, Khan S, Jain $U$, et al. A comparative evaluation of intravenous labetalol versus oral nifedipine for control of severe pregnancy-induced hypertension with low dose regimen. Int J Med Sci Public Health 2016;5(6):1183-6.

[10] Shekhar S, Sharma C, Thakur S, et al. Oral nifedipine or intravenous labetalol for hypertensive emergency in pregnancy: a randomized controlled trial. Obstet Gynecol 2013;122(5):1057-63.

[11] Magpie Trial Follow-Up Study Collaborative Group. The Magpie Trial: a randomised trial comparing magnesium sulphate with placebo for pre-eclampsia. Outcome for women at 2 years. BJOG 2007;114(3):300-9.

[12] Dhali B, Bhattacharya S, Ganguly RP, et al. A randomized trial of intravenous labetalol and oral nifedipine in severe pregnancy induced hypertension. Int J Reprod Contracept Obstet Gynecol 2012;1(1):42-6. 